\title{
Proceso de modelación en clase de Matemática
}

\author{
Modeling process in the Math class \\ O. Fernández-Sánchez iD , M. Angulo-Cruz iD
}

\begin{abstract}
- this article shows the resolution of an arithmetic problem through a process of modeling in the classroom as a proposal to accompany the student in the process of construction of mathematical knowledge. For this, phases analogous to those of a process of Mathematical modeling based on partial differential equations are proposed, it is used to understand the dynamics of a public health problem such as the spread of tuberculosis, which allows health authorities to take measures to control this disease. Five phases are identified in the process, which begin with an experiential situation and reach a symbolic phase, which is when the abstract model is found, a noumenon in terms of the philosopher Kant. This process allows the student to understand how a symbolic model such as the division algorithm emerges.
\end{abstract}

Index Terms - division, noumenon, mathematical modeling, phenomenon, teaching.

Resumen-Se pretende mostrar la resolución de un problema aritmético, mediante un proceso de modelación en el aula de clase, como una propuesta para acompañar al estudiante en el proceso de construcción de conocimiento matemático. Para esto, se proponen fases análogas a las de un proceso de modelación matemática basado en ecuaciones diferenciales parciales, usado para entender la dinámica de un problema de salud pública, como la diseminación de la tuberculosis, que les permite a las autoridades de salud tomar medidas para el control de esta enfermedad. Se identifican cinco fases, las cuales inician con una situación experiencial hasta llegar a una fase simbólica, que es cuando se encuentra el modelo abstracto, un nóumeno en términos del filósofo Kant. Este proceso, permite al estudiante entender como emerge un modelo simbólico como el algoritmo de la división.

Palabras claves - división, enseñanza, fenómeno, noúmeno, modelación matemática.

\section{INTRODUCCION}

$\mathrm{S}$ EGÚN Kant, en [1], "se halla en nuestro concepto que denominemos entes de los sentidos (phaenomena) a ciertos objetos a título de fenómenos, distinguiendo el modo como los intuimos de su constitución en sí, de suerte que, por esa última constitución, aunque no la contemplemos en ellos, los opongamos a aquellos por decirlo así, ellos o también otras

Este manuscrito fue enviado el 30 de noviembre de 2017 y aceptado el 26 de enero de 2019.

O. Fernández-Sánchez, Doctor en Ciencias de la Educación, es docente en el Departamento de Matemáticas, de la Universidad Tecnológica de Pereira, Pereira, Risaralda (Colombia). E-mail: oscarf@utp.edu.co cosas posibles que no sean objeto de nuestros sentidos, como objetos sólo pensados por el entendimiento y los denominemos entes del entendimiento (noumeno)" (p. 19).

Desde el punto de vista del aula de clase de matemáticas, los símbolos que componen el lenguaje de las matemáticas (en plural para referir no solo la matemática de la cultura de Occidente) y las relaciones que ellos simbolizan constituyen modelos relacionales generales que son productos culturales y como tales resultan de procesos de modelación que en muchos casos se pasa por alto, negando al estudiante la posibilidad de reconstruir y comprender el alcance y la fuerza de dichos modelos para sintetizar las dinámicas del entorno social, natural y cultural, es decir, al prescindir en el aula de clase, de la enseñanza de un objeto matemático a través de su devenir como modelo, se pierde gran parte de su valor como elemento cognitivo, y no solo esto, sino que se trata el modelo tan solo como un objeto del intelecto, carente de significado. Ya lo decía Kant en [1], que en el conocimiento de un objeto es necesaria la combinación entre el intelecto y lo sensible, puesto que al separarlos se producirían "intuiciones sin conceptos 0 conceptos sin intuiciones, y en ambos casos representaciones que no podemos referir a un objeto determinado" (p. 24). Así mismo, considera que "en cualquier principio, el uso trascendental de un concepto consiste en referirlo a las cosas en general y en sí mismas; el uso empírico, en referirlo sólo a fenómenos, es decir a objetos de una experiencia posible" (p. 12). Para ilustrar este hecho considera lo que sucede con los conceptos matemáticos, que, según él, los principios y la representación de los objetos en esta ciencia, "son producidos totalmente a priori en el espíritu" (p. 12); sin embargo, ellos carecerían de significado si no fuera posible exponer este último en fenómenos. Con esto expone la necesidad de llevar un concepto al campo de los sentidos, exponiendo en la intuición el objeto que le corresponde a dicho significado, a riesgo de dejar el objeto carente de significado. Asegura Kant en [1], que

La matemática cumple con este requisito construyendo la figura, que es un fenómeno presente a los sentidos, aunque producido a priori. En esa misma ciencia, el concepto de

M. Angulo-Cruz, Magister en Comunicación Educativa y Magister en Educación y Desarrollo Humano, es docente en el Departamento de Matemáticas, de la Universidad Tecnológica de Pereira, Pereira, Risaralda (Colombia).E-mail: monac@utp.edu.co 
magnitud busca también su apoyo y sentido en el número, y este en los dedos, en las bolas del tablero o en las rayas y puntos que se ponen ante los ojos. El concepto sigue siendo producido a priori y con él todos los principios sintéticos o fórmulas derivados de esos conceptos; pero el uso de los mismos y la referencia a supuestos objetos no pueden buscarse en definitiva más que en la experiencia (p. 12).

En un proceso de modelación matemática, se espera que se obtenga un modelo abstracto con el cual se puedan resolver una serie de situaciones problema con una cierta estructura relacionada con el modelo. Pero, si se indaga desde otros puntos de vista acerca del modelo y lo que éste representa, surgen varios interrogantes acerca de lo que se modela. Interrogantes sobre lo que se entiende por verdad, objetividad, realidad y objetividad. Sobre lo que es la verdad, Nietzsche en [2], dice que "solo mediante el olvido puede el hombre alguna vez además llegar a creer que él posee una "verdad"” (p. 25). Este autor, considera que cuando el hombre se encuentra con un objeto o hecho desconocido, usa palabras para designarlo, para en cierta forma poseer su esencia, y con el pasar del tiempo y del uso reiterado de dichas palabras, se olvida que estas son solo eso, palabras y se toman por las cosas mismas. Y concluye que, "las verdades son ilusiones de las cuales se ha olvidado que ellas lo son, metáforas que se han desgastado por el uso" (p. 30) y después de un tiempo de uso reiterado se van tornando en lenguaje literal, por ejemplo: memoria USB, puerto USB, autopista de la información, raíz cuadrada, triángulo isósceles, triángulo escaleno, por nombrar algunos, palabras que inicialmente se usaron para designar cosas y que hoy las generaciones más jóvenes las toman por verdades, desconociendo su uso metafórico inicial.

\section{A. Modelación matemática en salud pública}

Para ilustrar con un caso, donde el significado de los objetos matemáticos es fundamental, debido a la utilidad e influencia que este tiene en la vida de las personas, es el uso de modelos matemáticos para el entendimiento y control de enfermedades, la llamada Epidemiología Matemática, un área de investigación dentro de la Biomatemática, que busca entender la dinámica de enfermedades contagiosas como la tuberculosis, mediante modelos matemáticos que permitan orientar las medidas de control que puedan tomar las autoridades sanitarias. La tuberculosis, según la Organización Mundial de la Salud (OMS), es una enfermedad infecto contagiosa, causada por un bacilo llamado Mycobacterium tuberculosis, la cual afecta a billones de personas en el planeta cada año, no solo en los pulmones, como tradicionalmente se ha creído, pues también se presenta en órganos tan impensables como los del sistema óseo. En términos simples, una persona infectada con el bacilo y que ha llegado al estado sintomático, en el caso de la tuberculosis pulmonar, contagia a otra persona sana, y susceptible de contraerla, a través del aire cuando la primera tose o habla [3].

En el proceso de modelación en epidemiología se tiene en cuenta los siguientes aspectos: Reconocer una situación problema, definir las variables y sus relaciones, estructurar la situación problema de forma verbal, gráfica y simbólicamente en términos de las variables y sus relaciones, y finalmente validar la solución obtenida en términos de la situación.

\section{B. Modelación matemática en el aula}

La enseñanza de la matemática, es un tema de interés para la comunidad educativa, dada la importancia que ha adquirido el conocimiento de diferentes enfoques y metodologías que pueden ayudar a mejorar los procesos de enseñanza y aprendizaje de dicha ciencia. El lenguaje de la Matemática está compuesto por símbolos y relaciones, los cuales es posible usarlos para modelar fenómenos del entorno natural y social de las personas, los cuales constituyen una ayuda para entender e intervenir ese entorno en su propio beneficio. Así lo considera el MEN en [4] cuando señala que "la resolución de problemas en un amplio sentido se considera siempre en conexión con las aplicaciones y la modelación. La forma de describir ese juego o interrelación entre el mundo real y las matemáticas es la modelación" (p. 97).

Este hecho implica que, en el momento de enseñar matemáticas, es necesario permitir que el estudiante visualice la relación de la realidad con los modelos que conforman el lenguaje de la Matemática. El proceso de modelación es un medio por el cual el profesor puede lograr hacer significativo el aprendizaje de la matemática, a través de la resolución de la situación problema en un ambiente de aula con materiales didácticos que constituyen una situación real, como la situación presentada en este artículo, aunque en la mayoría de los casos, es necesario construir materiales, de modo que se haga una simulación de un fenómeno real, en el cual, haya la necesidad de resolver un problema asociado. Estos aspectos, se evidencian cuando el estudiante llega a posibles resultados y logra validarlos con la situación problema planteada al inicio del proceso; así mismo, puede describir las cantidades resultantes y sus relaciones, también, cuando el estudiante es capaz de plasmar en el cuaderno una aproximación esquemática del fenómeno haciendo uso de símbolos para etiquetar cada elemento involucrado en la resolución del problema [5].

Hay un aspecto clave, cuando se opta por la modelación en el aula de clase de matemáticas y es que, el profesor debe trascender de su papel de enseñante para convertirse en acompañante del proceso. Claro está que, es vital su experticia respecto al conocimiento, cuando es él quien prepara, diseña y construye los materiales que conforman el ambiente didáctico que requieren los estudiantes en el desarrollo del proceso de modelación matemática [5].

Según [6], en el ambiente social del aula de clase se da una serie de interacciones a nivel de pares, entre estudiantes, alrededor del objeto matemático involucrado en la situación problema planteada, en las cuales la intervención explicita del profesor puede o no darse. Así mismo, señala que el estudiante se motiva a contribuir activamente en la construcción del modelo matemático con "aportes productivos, que agregan valor a lo que se discute, que ayudan a otros a expresar lo que piensan" (p. 23). De modo que si el profesor permite al 
estudiante hacer parte activa en la construcción del conocimiento en el marco de la modelación matemática como propuesta didáctica, "su participación se caracteriza por las interacciones y las contribuciones que se asumen como elementos que ayudan a construir las comprensiones necesarias para el aprendizaje en el discurso matemático de aula" (ídem). Esta es una propuesta que, favorece la visión de un profesor que acompaña al estudiante en la construcción activa de su conocimiento matemático y no solo enseña a unos estudiantes que, de manera pasiva, al estilo tradicional, solo observan y llevan a su cuaderno todo aquello que a veces aceleradamente el profesor escribe en el tablero, sin hacerle inventario a unos contenidos que, a veces se tornan áridos para el mismo profesor.

\section{PROCESO DE MODELACIÓN EN SALUD PÚBLICA}

\section{A. Situación problema}

Entender mediante una simulación con un modelo matemático las tendencias de la infección tuberculosa en la zona urbana de la ciudad de Cali, a partir de datos tomados durante los años 1990-1999 por la Secretaría Municipal de Salud de Cali [3].

\section{B. Fase 1: reconocimiento de variables y relaciones}

Para generar el modelo, se considera un índice conocido como Riesgo Anual de Infección (RAI) el cual (Styblo, 1991, citado en [3], p. 38) lo define como «la probabilidad que tiene un individuo, quien no ha estado previamente en contacto con el bacilo tuberculoso, de infectarse en el curso de un período relativamente corto, tal como un año». Según (Styblo, 1991, citado en [3], p. 38), este índice sintetiza factores de riesgo de contraer la infección como: intensidad, frecuencia y duración de contacto con un infectado sintomático, estado de salud del huésped, así como características del agente infeccioso.

\section{Fase 2: estructuración de la situación problema en términos de las variables y sus relaciones}

Según [3], para estructurar la dinámica latente en la situación problema se consideran la variable tiempo, $t$, y la variable edad, a, las cuales constituyen las variables independientes del modelo. Las variables dependientes se generan de las subpoblaciones determinadas por la dinámica de la enfermedad: susceptibles, (S), como las personas que no han sido infectadas con el bacilo; la subpoblación de protegidos por la vacuna $\mathrm{BCG}$, (P); la subpoblación de personas que han sido infectados, pero que no son sintomáticos, llamados infectados latentes, (I); los que al aplicarles una prueba diagnóstica conocida como "frotis $\mathrm{BK}$ ", resultan positivos, $(\mathrm{BK}+)$, por lo cual son infectados, sintomáticos y por tanto contagiosos, llamados casos abiertos, (A), y los negativos al frotis (BK-), lo que significa que están enfermos pero que por estar en tratamiento han dejado de ser infecciosos, considerados por esto casos no abiertos, (NA); la subpoblación de los curados, también llamada de los removidos, (R), y por último está la subpoblación de los enfermos que abandonan el tratamiento y el bacilo hace mutación, volviéndose resistente a los medicamentos ya aplicados, considerados crónicos, (C). Para cada clase se considera una función de densidad de probabilidad de pertenecer a ella, dependiente de la edad $a$ y del tiempo $t$, simbolizada por $\mathrm{S}(a, t), \mathrm{P}(a, t), \mathrm{I}(a, t), \mathrm{A}(a, t), \mathrm{NA}(a, t), \mathrm{C}(a, t)$ y $\mathrm{R}(a, t)$ respectivamente.

\section{Fase 3: estructuración gráfica de la situación problema en términos de las variables y sus relaciones}

Para esta fase [3] considera que una vez definidas las variables respecto a la situación problema se traza un diagrama que permita visualizar la forma como las personas hacen tránsito entre las subpoblaciones generadas por la dinámica de la enfermedad. Para esto se introduce una función $b(a)$ que determina la tasa de contacto entre infecciosos y grupos de susceptibles. Se supone que todos los recién nacidos son susceptibles y que la transferencia de infección es debida a la interacción entre el susceptible y el infeccioso, el infectado entra en un período largo de latencia y después pasa a la clase de infecciosos o casos abiertos.

También se considera la función $\lambda(t)$, la cual representa la fuerza de infección de la enfermedad. En el sentido que la infecciosidad de muchos individuos infectantes se unen para crear una fuerza que desplaza a otros individuos del estado susceptible. Al ser mayor la fuerza de infección, más temprano los susceptibles son infectados y más rápido decrece la fracción de susceptibles al estado infectado. Se simboliza como $\gamma_{1}$ y $\gamma_{2}$ a las fracciones de individuos infectados que pasan a las clases de abiertos (A) y no abiertos (NA) respectivamente. Las tasas de transición entre las diferentes clases están dadas por $k_{i}, i=1$, $\cdots, 6, \mu(a)$ es la tasa de mortalidad.

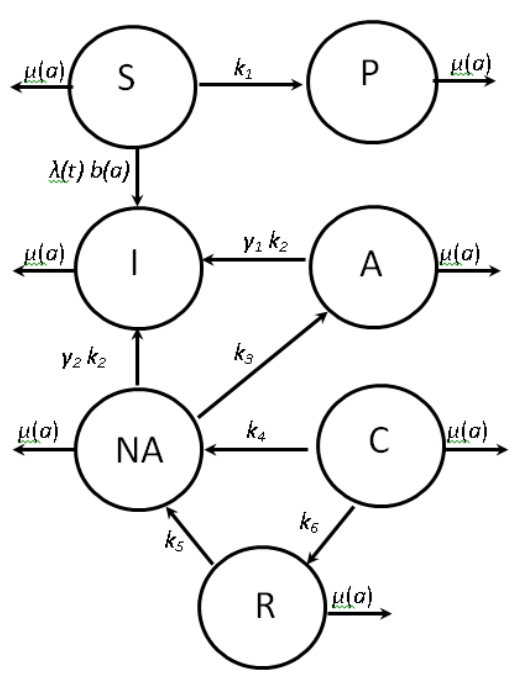

Fig. 1. Aproximación gráfica de la dinámica de transmisión de la infección tuberculosa entre las poblaciones implicadas [3].

\section{E. Fase 4: estructuración simbólica de la situación problema en términos de las variables y sus relaciones}

El modelo epidemiológico estructurado en la edad en [3] (p. 40), es un modelo matemático definido mediante las siguientes siete ecuaciones diferenciales parciales: 


$$
\begin{aligned}
\frac{\partial S(a, t)}{\partial a}+\frac{\partial S(a, t)}{\partial t} & =-\lambda(t) b(a) S(a, t)-\mu(a) S(a, t)+k_{1} P(a, t) \\
\frac{\partial P(a, t)}{\partial a}+\frac{\partial P(a, t)}{\partial t}= & -k_{1} P(a, t)-\mu(a) P(a, t) \\
\frac{\partial I(a, t)}{\partial a}+\frac{\partial I(a, t)}{\partial t}= & =\lambda(t) b(a) S(a, t)-k_{2} I(a, t)-\mu(a) I(a, t) \\
\frac{\partial A(a, t)}{\partial a}+\frac{\partial A(a, t)}{\partial t}= & \gamma_{1} k_{2} I(a, t)-k_{3} A(a, t)-\mu(a) A(a, t) \\
\frac{\partial N A(a, t)}{\partial a}+\frac{\partial N A(a, t)}{\partial t}= & \gamma_{2} k_{2} I(a, t)+k_{3} A(a, t)-k_{5} N A(a, t) \\
& -k_{4} N A(a, t)-\mu(a) N A(a, t) \\
\frac{\partial C(a, t)}{\partial a}+\frac{\partial C(a, t)}{\partial t}= & k_{4} N A(a, t)-k_{6} C(a, t)-\mu(a) C(a, t) \\
\frac{\partial R(a, t)}{\partial a}+\frac{\partial R(a, t)}{\partial t}= & k_{5} N A(a, t)+k_{6} C(a, t)-\mu(a) R(a, t),
\end{aligned}
$$

Fig. 2. Modelo epidemiológico estructurado en la edad. Fuente: [3] (pg. 40).

\section{F. Fase 5: validación e interpretación del modelo en términos de la situación problema}

Este tipo de sistemas de ecuaciones diferenciales se resuelven de manera aproximada mediante métodos numéricos y un software matemático como MatLab, se dice entonces que la solución del modelo es numérica. Para el uso del software se escribe un programa y se interpreta la salida en términos de la situación problema. La salida se obtiene de forma gráfica, como en Fig. 3 y Fig. 4, de las cuales se deduce, por ejemplo, de la Fig. 3 que "la cantidad de nuevos casos es mayor entre los $35 \mathrm{y}$ 50 años" y en la Fig. 4, que

el índice agregado de infección presenta su punto más alto a la edad de 30 años, pero presenta un porcentaje muy alto, por encima del $0.8 \%$ entre los 20 y 40 años, una situación que debe preocupar a las autoridades de salud pues indica que de cien mil personas en Cali al menos 800 están infectadas con tuberculosis [13], (p. 55).

\section{G. Utilidad de los modelos en ciencia}

Los modelos matemáticos en ciencias como la Epidemiología, se utilizan para entender la forma en que ciertas enfermedades, como la tuberculosis pulmonar, de tipo infectocontagiosas se diseminan entre la población, y poder exponer ante las autoridades sanitarias la necesidad de tomar acciones de prevención y control de estas enfermedades.

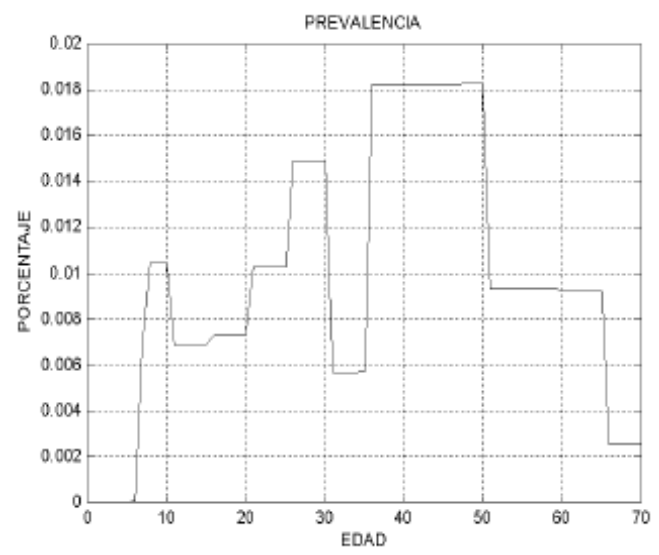

Fig. 3. Prevalencia de la enfermedad por edades. [3], p. 54.

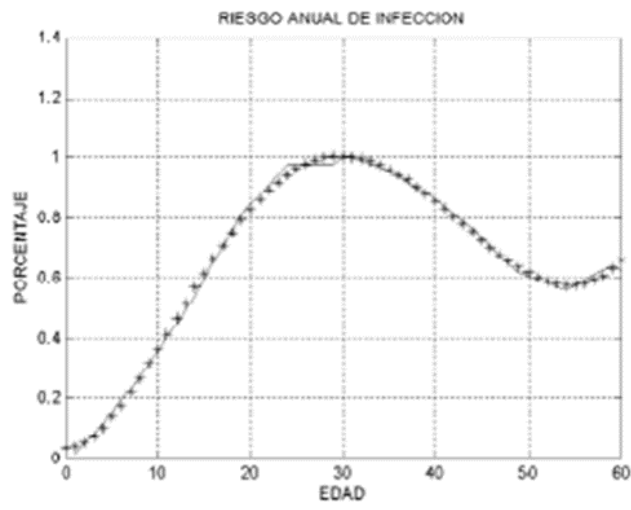

Fig. 4. Estimaciones del RAI para los grupos de edad entre 0 y 60 años, este índice refleja la carga de infección tuberculosa en una comunidad. [3], p. 55

Ahora se procede a estructurar, en un proceso análogo, una situación problema en el aula de clase de matemáticas, en el área de la aritmética. Específicamente, se mostrará cómo es posible abordar el algoritmo de la división, como el resultado de un proceso de modelación, a partir de una situación problema de repartición de unas golosinas entre varios niños.

\section{PROCESO DE MODELACIÓN EN EL AULA DE CLASE}

Para el caso del aula de clase se considera una situación problema en una clase de aritmética de nivel tercero de primaria. En este nivel se cuenta con ciertos saberes en aritmética como son: contar, formar conjuntos de objetos, sumar cantidades de objetos, restar de una cantidad de objetos cierta cantidad menor de objetos, multiplicar cierta cantidad de objetos un número de veces (como un proceso de repetición y luego reunión).

Teniendo en cuenta estos saberes, se plantea una situación problema, con el objetivo de acompañar a los estudiantes en su proceso de aprendizaje del concepto de división desde la dupla proceso/producto, donde el producto es el concepto de cociente, generado como resultado de un proceso de modelación matemática.

\section{A. La situación problema}

¿Cómo repartir 9 turrones de coco entre 4 niños sin partir ningún turrón y de modo que a cada niño le corresponda la misma cantidad de turrones? [5].

Se propone para la resolución de esta situación tener en cuenta las fases del proceso de modelación matemático planteado antes en el caso de la diseminación de la infección tuberculosa en la zona urbana de la ciudad de Cali, en el periodo 1990-1999. Se tienen entonces las siguientes fases para el problema de la división en el aula de clase:

\section{B. Fase 1: reconocimiento de cantidades y relaciones mediante manipulación}

Inicialmente se forman grupos de 4 estudiantes y se les entrega 9 turrones de coco a cada grupo. Se plantea la situación problema y se les invita a resolverla usando los saberes previos. Se les pregunta por las posibilidades de solución de la situación, 
así como las cantidades involucradas y sus posibles relaciones desde los conceptos previos que maneja el grupo.

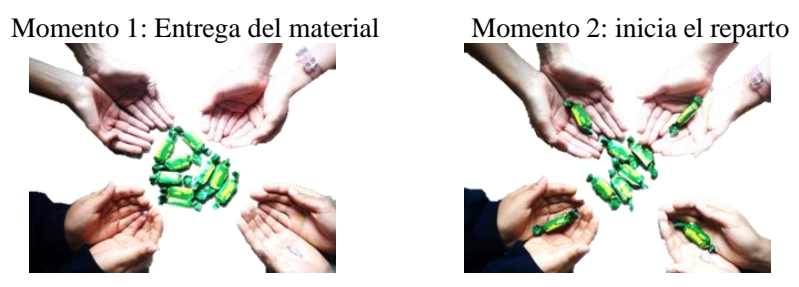

Momento 3: finaliza el reparto

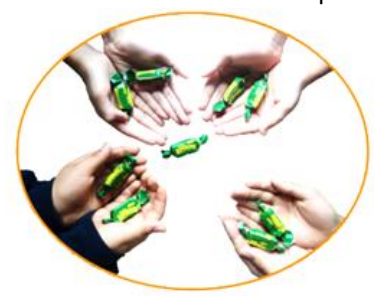

Fig. 5. Momentos en la fase de manipulación del proceso de modelación del algoritmo de la división [5].

Esta es una fase de pensamiento concreto en la cual se manipula material didáctico, que para la situación presente ya está disponible en almacenes de cadena, aunque no siempre es así, pues a veces es necesario que sea diseñado y construido por el docente, con el fin de relacionar al estudiante con un fenómeno determinado que le permita, mediante el uso de sus sentidos generar un conocimiento emergente, acorde con los objetivos que el docente ha planteado para la clase. En esta fase, los estudiantes generan cantidades que serán relacionadas en una fase posterior con símbolos como numerales y de las operaciones como suma, resta y producto.

C. Fase 2: estructuración verbal de la situación problema en términos de las variables y sus relaciones:

Una vez que cada grupo ha reconocido las cantidades involucradas en la situación problema, se invita a describir, mediante lenguaje matemático verbal, las cantidades resultantes, su naturaleza y las relaciones entre ellas surgidas de la manipulación que cada grupo realizó del material didáctico.

Esta actividad permite, en la fase siguiente, mediante un proceso de graficación, plasmar en los cuadernos de cada estudiante, una aproximación esquemática del fenómeno, en la cual se empieza a usar los símbolos para etiquetar cada elemento involucrado en la resolución de la situación.

\section{Fase 3: estructuración gráfica de la situación problema en términos de las variables y sus relaciones}

Una vez cada grupo hace la descripción del fenómeno usando lenguaje matemático verbal, se busca aproximar mediante un gráfico la resolución de la situación. Esta es una fase intermedia entre un fenómeno determinado por la manipulación de objetos observables y tangibles y un modelo simbólico abstracto. En esta fase, ya se puede hablar de una aproximación previa ya que un gráfico no es precisamente el fenómeno, sino una aproximación de éste, aunque no es aun el modelo matemático que se busca para representar el proceso de la división. Se siguen los siguientes pasos:

Paso 1: Graficar de una manera aproximada (desde el punto de vista de cada grupo de estudiantes) la situación final de la fase de manipulación (ver momento 3, Fig. 5), después de repartir los turrones con las condiciones dadas, y que cada niño quedó con una cantidad de turrones y sobró una cantidad, luego de la repartición.

Enseguida se ilustra, en la Fig. 6, una aproximación a la gráfica que en diferentes ocasiones los grupos de estudiantes en [5] han realizado.

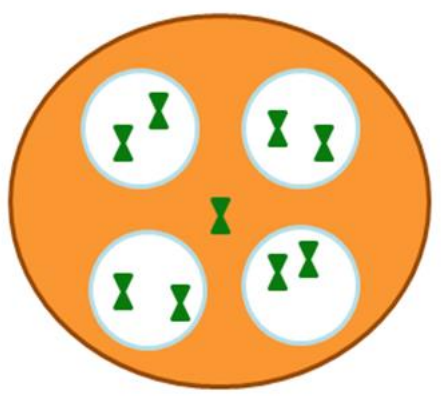

Fig. 6. Representación gráfica del momento 3 en la repartición de los nueve turrones entre los cuatro niños [5].

Paso 2: Una vez que los estudiantes han reconocido cada elemento involucrado en el fenómeno con el material didáctico, se simplifica la gráfica usando puntos en lugar de dibujos de los turrones de modo que se obtenga un gráfico con un grado mayor de generalidad. Luego se etiquetan las cantidades que aparecen con cifras numerales, que en este caso son $9,8,2$ y 1 , como en la Fig. 7. El numeral 9 para indicar la cantidad de turrones que habían inicialmente, el numeral 8 para indicar la cantidad de turrones repartidos, el numeral 2 para indicar la cantidad de turrones que corresponde a cada niño y el 1 para indicar el turrón que sobró.

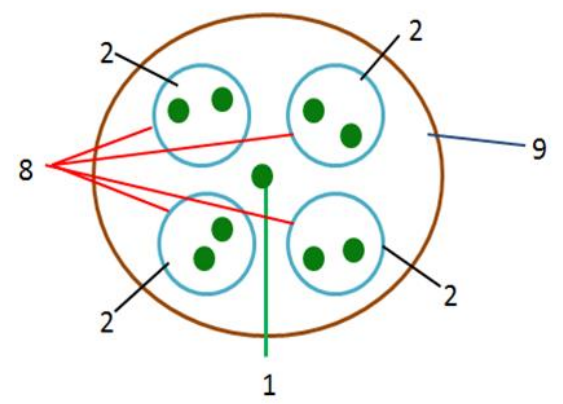

Fig. 7. Representación gráfica simplificada del momento 3 en la repartición de los nueve turrones entre los cuatro niños, usando puntos y cifras numerales [65].

\section{E. Fase 4: estructuración simbólica de la situación problema en términos de las cantidades y sus relaciones:}

Después de la acción de repartir los turrones entre los niños, en el proceso se distinguen 5 cantidades, etiquetadas junto a sus 
numerales, los cuales se relacionan entre sí, según se indica en la Tabla I:

TABLA I

ETIQUETAS Y RELACIONES ENTRE LAS CANTIDADES

\begin{tabular}{lll}
\hline \hline Cantidad de objetos & \multicolumn{1}{c}{ Tipo de relación } & Cantidad de niños \\
\hline 9 turrones... & $\begin{array}{l}\text { disponibles para } \\
\text { repartir entre... }\end{array}$ & 4 niños \\
2 turrones... & $\begin{array}{l}\text { recibe... } \\
\text { son repartidos entre el } \\
\text { total de los... }\end{array}$ & 4 niño \\
8 turrones... & $\begin{array}{l}\text { sobra después de la } \\
\text { repartición }\end{array}$ & \\
1 turrón & & \\
& & \\
\hline \hline
\end{tabular}

Estas relaciones entre las cantidades, para este caso particular, se acomodan de la siguiente manera, según la sintaxis del lenguaje de la Matemática de Occidente:

\begin{tabular}{c|l}
9 turrones & 4 niños \\
\cline { 3 - 3 } (menos) 8 turrones & 2 turrones (para cada niño)
\end{tabular}

(sobra) 1 turrón

Fig. 8. Arreglo estándar para representar los datos y sus relaciones en la Tabla I sugerido en [5].

Este arreglo particular sin (las etiquetas nominales) se puede ajustar a cualquier situación en la cual se usen las mismas cantidades, pero con agentes distintos. Por ejemplo, ¿cómo empacar 9 toneladas de harina (o cualquier otra sustancia o material) en 4 bolsas (o cajas o cualquier otro tipo de empaque) sabiendo que la capacidad de cada empaque es de 2 toneladas, y cada uno debe estar lleno en toda su capacidad? Así como este, se pueden encontrar en la cotidianidad, y en múltiples oficios, casos donde, con este arreglo, es posible resolver la situación problema. Por este hecho, es necesario, y pensando en la economía del lenguaje, abreviar el arreglo en un modelo simplificado donde las etiquetas que lo particularizan desaparecen y solo quedan los numerales que indican las cantidades señaladas:

\begin{tabular}{c|c}
9 & 4 \\
-8 & 2 \\
\hline 1 &
\end{tabular}

\section{F. Fase 5: generalización simbólica de la solución en términos de las cantidades y sus relaciones:}

En este punto, es posible hablar de un modelo relacional generalizado, es decir, se ha pasado de un fenómeno, cuando los estudiantes por medio de sus sentidos reconocieron y resolvieron la situación problema, a un nóumeno, cuando junto al profesor, los estudiantes puedan llegar a reconocer y entender el modelo matemático generalizado mediante el cual se relacionan cinco cantidades: $\mathrm{m}, \mathrm{n}, \mathrm{q}, \mathrm{p}, \mathrm{r}$. El numeral $\mathrm{m}$, representa la cantidad a repartir; $n$, la cantidad entre la que se reparte m; q, lo que corresponde a cada n; p, la cantidad repartida y $\mathrm{r}$ la cantidad que sobra después de repartir. El modelo matemático general quedaría así

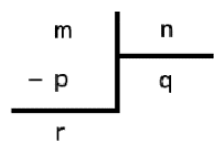

En este modelo, cada elemento recibe una denominación en el lenguaje de la matemática en la cultura de Occidente. Al elemento $\mathrm{m}$ se le denomina dividendo, al elemento $\mathrm{n}$, divisor, al elemento q, se le denomina cociente y al elemento r, residuo.

\section{G. Fase 6: validación del modelo en términos de la situación problema:}

Una vez llegados al modelo matemático que relaciona las cantidades mencionadas simbólicamente, se confronta cada una con la cantidad inicial de turrones, antes de repartirlos a los niños, la cantidad que cada niño recibió en la acción de la repartición, así como el turrón que sobró. Se busca que los niños puedan relacionar el fenómeno ocurrido en la repartición, en la fase de manipulación, con el nóumeno obtenido en la fase de simbolización.

\section{CONCLUSIONES}

En la Fig. 9, se muestra un esquema del proceso de modelación que se ha definido por las fases anteriores, el cual indica que éste inicia con una situación problema (fenómeno), pasa a través de una manipulación de los elementos que componen la situación, una graficación de las posibles relaciones de esos elementos, como una aproximación inicial, en la cual se vislumbra un primer nivel de abstracción de las cantidades, las variables y las relaciones que se hacen evidentes en un ambiente de conocimiento de los saberes previos por parte de los estudiantes.

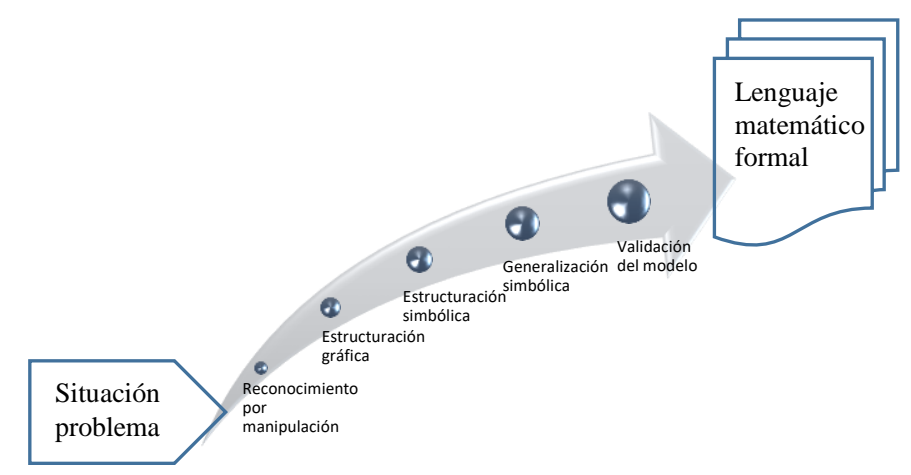

Fig. 9. Esquema del proceso de modelación matemático en el aula [65].

Es en la gráfica donde se empiezan a plasmar los nombres de las variables, a señalar las cantidades que intervienen en la situación y el proceso que los grupos de estudiantes están siguiendo para resolverla. Con el acompañamiento del profesor, se llega hasta un modelo matemático generalizado y abstracto (nóumeno), el cual va a entrar a formar parte del repertorio de modelos que el estudiante ya conoce. Junto con los modelos ya conocidos (saberes previos) y los modelos encontrados en la resolución de la situación planteada (saberes emergentes), se busca que el estudiante comprenda y aprehenda el hecho 
epistemológico del conocimiento matemático, paralelamente va ampliando el conocimiento del "léxico" que compone el lenguaje de la Matemática. Un lenguaje que, en primera instancia, se muestra abstracto y en ocasiones, un poco árido, pero que, en su devenir histórico epistemológico, muestra su utilidad y versatilidad como instrumento para entender el entorno natural, cultural y social, en la medida que es un componente esencial para resolver problemas relacionados con dichos entornos.

Es muy importante que, para la fase inicial concreta, se planee sobre el conocimiento profundo del tema matemático a trabajar, y desde este conocimiento se prepare y diseñe el material didáctico a manipular por parte de los estudiantes. Este material debe ser coherente con la temática en la cual está enmarcada la situación problema, puesto que de su manipulación emergerán elementos que serán claves para obtener las cantidades constantes, las variables y las relaciones que componen el modelo matemático emergente. Este modelo se convierte en herramienta con la cual se obtendrán, en un próximo proceso, otros modelos cuyo nivel de complejidad será cada vez mayor, a medida que el estudiante avance en el conocimiento de la Matemática.

\section{REFERENCIAS}

[1] I. Kant, Critica de la razón pura. Dialéctica trascendental y metodología trascendental. Tomo II. Traducción de José Rovira Armengol. Bogotá: Universales.

[2] F. Nietzsche, Sobre Verdad y Mentira en Sentido Extramoral. Traducción Jorge Castillo. Bogotá, 2006.

[3] E. De la Pava, O. Fernández y B. Salguero, "Modelación matemática con estructura de edad del riesgo de infección tuberculosa en la ciudad de Cali”, revista Matemática: Enseñanza universitaria. Escuela Regional de Matemática. Universidad del Valle, vol. XVI, No. 2, pp. 37-56, 2008.

[4] Ministerio de Educación Nacional, MEN. Lineamientos Curriculares Matemáticas. Áreas obligatorias y fundamentales. Bogotá: Cooperativa Editorial Magisterio, 1998.

[5] O. Fernández, "El proceso de modelación en el aula de clase de matemáticas" presentada en la Maestría en Enseñanza de la Matemática., Universidad Tecnológica de Pereira, Pereira, 2016.

[6] J. Villa, "Interacciones y contribuciones. Formas de participación de estudiantes de quinto grado en ambientes de modelación matemática", Revista Actualidades Investigativas en Educación. Instituto de Investigación en Educación, INIE, Universidad de Costa Rica, vol. 16, no. 3, pp. 1-27, 2016.

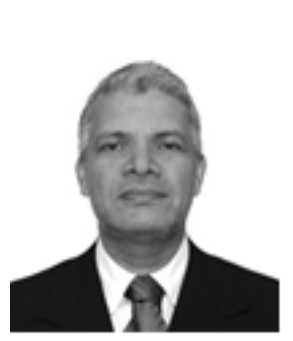

Oscar Fernández-Sánchez nació en Popayán, Cauca, el 3 de abril de 1964. Licenciado en Educación en la Especialidad Matemáticas, Universidad del Cauca. Magister en Ciencias Matemáticas, Universidad de Valle, Cali. Doctor en Ciencias de la Educación, RUDECOLOMBIA-UTP, Pereira.

El profesor Fernández-Sánchez es Profesor Titular de planta del Departamento de Matemáticas en la Universidad Tecnológica de Pereira. Entre sus campos de interés están la Etnomatemática, Teoría Cognitiva de la Matemática, Modelación Matemática en el aula y Didáctica de la Matemática.

Entre sus publicaciones están: O. Fernández, F. Mesa y A. Valencia, Introducción al Álgebra Lineal, Bogotá, D.C., Ediciones ECOE, 2012. O. Fernández, E. De la Pava y B. Salguero, "Modelación Matemática con estructura de edad del riesgo de infección tuberculosa en la ciudad de Cali," Matemáticas: Enseñanza Universitaria, Corporación Escuela Regional de Matemáticas, vol. XVI, no. 2, pp. 37-56, dic. 2008. O. Fernández, "Pensamiento matemático de los Mayas. Una creación metafórica, ” Entre Ciencia e Ingeniería, vol. 4, no. 8, pp. 174-188, dic. 2010.

El profesor Fernández es líder del Grupo de Investigación en Pensamiento Matemático y Comunicación-GIPEMAC. Ejerció como director de la Maestría en Enseñanza de la Matemática de la Universidad Tecnológica de Pereira, 2006-2012.

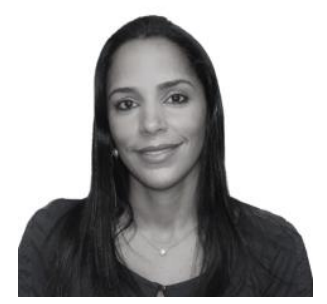

Mónica Angulo-Cruz nació en Cali, Valle, el 9 de marzo de 1976. Licenciada en Educación, Universidad San Buenaventura, Cali, Licenciada en Matemáticas, Universidad Nacional Abierta y a Distancia, Magister en Comunicación Educativa, Universidad Tecnológica de Pereira, Magister en Educación y Desarrollo Humano, Universidad San Buenaventura, Cali.

La profesora Angulo-Cruz es Profesora Titular de Tiempo Completo Transitoria en el Departamento de Matemáticas en la Universidad Tecnológica Pereira. Entre sus campos de interés están: Formación de profesores de Matemática, Teoría Comunicacional y Emocional de la Matemática.

Entre sus publicaciones están: M. Angulo, O. Fernández y F. Mesa, Formación de profesores de Matemática. Una experiencia desde la práctica educativa en el aula. Bogotá, D. C., Ediciones ECOE, 2012. M. Angulo, "La influencia de la comunicación educativa en la enseñanza de la matemática 
escolar, una mirada desde la teoría de Charles Sanders Pierce,"

Revista Ciencia y Técnica, vol. 18. No. 4, pp. 703-711, 2013.

M. Angulo y M. Castañeda, "Pensamiento matemático en ambientes no formales: un caso de investigación en Etnomatemática," Revista Ciencia y Técnica, vol. 2. No. 51, pp. 216-221, 2012.

La profesora Angulo-Cruz es coinvestigadora del Grupo de Investigación en Pensamiento Matemático y ComunicaciónGIPEMAC. 